\title{
All-Inorganic Red-Light Emitting Diodes Based on Silicon Quantum Dots
}

\author{
Batu Ghosh $1,2, *$ and Naoto Shirahata $2,3,4, *$ (D) \\ 1 Department of Physics, Triveni Devi Bhalotia College, Raniganj, West Bengal 713383, India \\ 2 International Center for Materials Nanoarchitectonics (MANA), National Institute for Materials \\ Science (NIMS), 1-1 Namiki, Tsukuba 305-0044, Japan \\ 3 Graduate School of Chemical Sciences and Engineering, Hokkaido University, Sapporo 060-0814, Japan \\ 4 Department of Physics, Chuo University, 1-13-27 Kasuga, Bunkyo, Tokyo 112-8551, Japan \\ * Correspondence: ghosh.batu@gmail.com (B.G.); Shirahata.naoto@nims.go.jp (N.S.)
}

Received: 25 June 2019; Accepted: 25 July 2019; Published: 26 July 2019

check for updates

\begin{abstract}
We report herein an all-inorganic quantum dot light emitting diode (QLED) where an optically active layer of crystalline silicon $(\mathrm{Si})$ is mounted. The prototype Si-QLED has an inverted device architecture of ITO/ZnO/QD/ $\mathrm{WO}_{3} / \mathrm{Al}$ multilayer, which was prepared by a facile solution process. The QLED shows a red electroluminescence, an external quantum efficiency (EQE) of $0.25 \%$, and luminance of $1400 \mathrm{~cd} / \mathrm{m}^{2}$. The device performance stability has been investigated when the device faces different humidity conditions without any encapsulation. The advantage of using all inorganic layers is reflected in stable EQE even after prolonged exposure to harsh conditions.
\end{abstract}

Keywords: light emitting diode; quantum dots; silicon; solution-process; all-inorganic device

\section{Introduction}

Silicon quantum dots (Si QDs), promising for applications in fluorescence biomarkers because of their low cytotoxicity [1-3], have recently shown improved device performance for prototype light emitting diodes (QLEDs) [4-8]. Being an indirect band gap semiconductor, silicon has poor optical properties (absorbance and emission), so an optically-excited type of LED is not a good option for use. However, we expect a dramatically improved device performance for a current-driven LED with Si QDs because external quantum efficiency (EQE) should be dominated by recombination rate between electrons and holes injected from electrodes. In fact, the best value of EQE for Si-QLEDs is as high as $8.6 \%$, which is still a record value in cadmium-free QLEDs $[4,9,10]$. The next important step is the development of an advanced Si-QLED, in addition to achieving an optimized optical performance in terms of low turn-on voltage, high EQE, strong brightness, Gaussian-shaped electroluminescence (EL) spectra, narrow emission band tunable over a wide range from visible to near-infrared (NIR) wavelengths, a long device lifetime, resistance characteristics against humidity and oxidation, no parasitic emission, and spectral stability over a broad range of operation voltages.

In typical QLEDs, a conventional device architecture is used. Indium tin oxide (ITO) is adopted for the anode, while aluminum (Al) is used for the cathode [11]. QLEDs have a multilayer structure that consists of an electron injection layer (EIL), an electron transportation layer (ETL), an active layer, a hole transportation layer (HTL), and a hole injection layer (HIL). For Si-QLEDs, polyethylene dioxythiophene:polystyrene sulfonate (PEDOT:PSS) and poly TPD serve as the HTL/HIL, while 1,3,5-tris(N-phenylbenzimidazol-2-yl)benzene (TPBi) is usually used as the EIL/ETL. However, there are papers reporting that an organic/inorganic hybrid multilayered structure might exhibit a poor stability against oxygen and moisture in ambient air [12]. It is also reported that the performance of the hybrid devices degrades over time. The degradation is induced by PEDOT:PSS, leading to the 
acid corrosion of ITO [13,14]. Maier-Flaig et al. studied morphological and compositional changes of Si-QLEDs during device operation [15]. For this purpose, they employed a combined analysis of cross-sectional transmission electron microscopy (TEM), energy filtered TEM, and energy dispersive X-ray spectroscopic mapping. Their TEM observation revealed that operation of a QLED with a conventional device structure at high applied voltage accelerated the significant morphological changes. Specifically, a strong electro-migration of QDs into the neighboring compositional organic EIL/ETL caused the formation of a large amount of macroscopic defects, leading to the collapse of multilayered structure. As predicted, such an intermixing effect spoiled the hole-blocking performance of the EIL/ETL, leading to the degradation of optical performance. To overcome these shortcomings, scientists have tried replacing the organic layers with inorganic layers to develop QLEDs that work even in a high operation voltage range [16-18]. A variety of metal oxides including zinc oxide (ZnO), nickel oxide $(\mathrm{NiO})$, tungsten oxide $\left(\mathrm{WO}_{3}\right)$, and molybdenum oxide $\left(\mathrm{MoO}_{3}\right)$ have been used till now for carrier injection and transportation layers, resulting in the fabrication of a device that survives for a long operation time even in harsh environments [19]. More recently, we reported that inverted device structure allows the Si-QLED to demonstrate a stable optical performance even in high electronic fields, in addition to the long-life device stability [20]. We concluded that the device degradation originating from the intermixing effect could be evitable when the inverted structure is adopted, although the multilayer still contains organic HTL/HIL of 4,4'-bis(carbazole-9-yl)biphenyl (CBP) molecules.

In this study, we report an all-inorganic Si-QLED for the first time. Except for the electrode, the multilayer demonstrated here was fabricated by spin-coating of colloidal inks of nanocrystals including Si QDs.

\section{Materials and Methods}

\subsection{Materials}

Triethoxysilane (TES) was purchased from TCI Chemicals (Tokyo, Japan). Electronic grade hydrofluoric acid (49\% aqueous solution) was purchased from Kanto Chemical (Tokyo, Japan). HPLC-grade toluene, isopropanol (IPA), ethanol, methanol acetone, ammonia solution $\left(\mathrm{NH}_{4} \mathrm{OH}\right.$, $28-30 \%)$, and hydrogen peroxide $\left(\mathrm{H}_{2} \mathrm{O}_{2}\right)$, were purchased from Wako Chemicals (Tokyo, Japan). 1-decene, zinc acetate dehydrate, and $\mathrm{WO}_{3}$ nanoparticle ink ( $2.5 \mathrm{wt}$. \% in IPA) were purchased from Sigma-Aldrich (St. Louis, MI, USA), and used as received.

\subsection{Preparation of Decane-terminated Si QDs}

In accordance with our previous protocol [21], hydrogen-terminated Si QDs were prepared by thermal disproportionation of hydrogen silsesquioxane derived from triethoxysilane, followed by hydrofluoric etching. The hydrogen-silicon surface bonds were replaced by carbon-silicon bonds through thermal hydrosilylation of 1-decene. We observed the enhanced photoluminescence quantum yields (PL QYs) from $0.2 \%$ (for hydrogen-termination) to $18 \%$ (for decane-termination) as a result of preventing the formation of nonradiative recombination channels in the surface of the QDs [22]. We call this role of the alkyl monolayer on enhanced PL QY the "anchor effect" [23]. XRD spectra of the as-prepared Si QDs are shown in Figure 1a. The peaks at $28^{\circ}, 47^{\circ}$, and $56^{\circ}$ were assigned as the (111), (220), and (311) planes of diamond cubic silicon. 

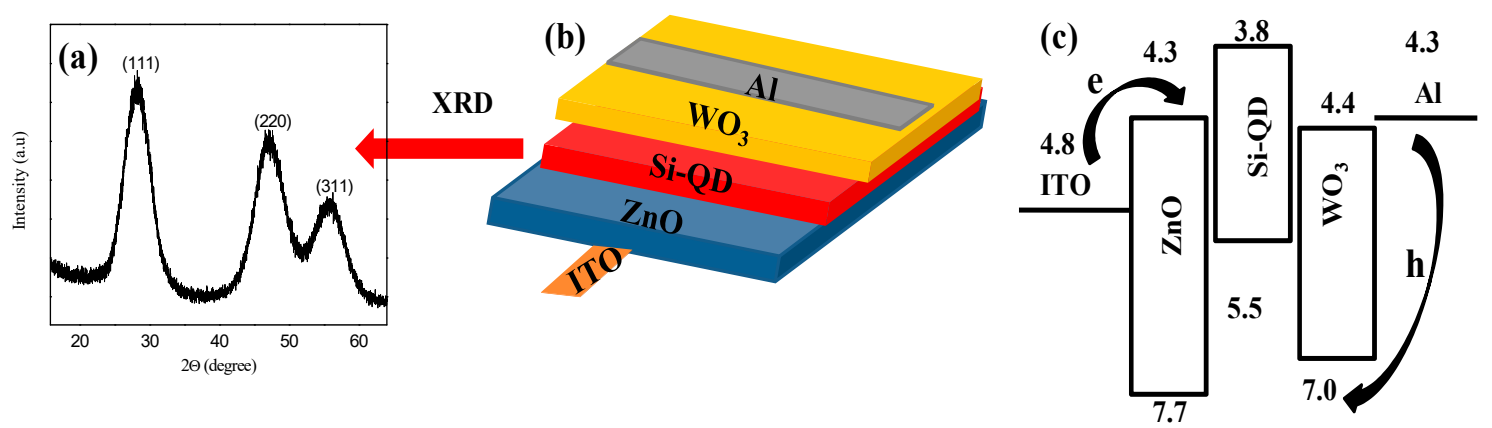

Figure 1. (a) X-ray powder diffraction pattern of Si QD, (b) a schematic representation of the red-light emitting Si QLED with an inverted device structure, and (c) the corresponding flat band diagram.

\subsection{Fabrication of the Si-QLED}

The Si-QLED was prepared on an ITO-coated glass substrate (sheet resistivity $~ 15 \Omega / \mathrm{sq}$ ). The substrate was etched in a thin strip and then cleaned by the standard RCA cleaning method which is widely used in the semiconductor industry. Then, 5 parts of Milli-Q water $(18.2 \mathrm{M} \Omega \cdot \mathrm{cm}, 50 \mathrm{~mL})$ were mixed with 1 part of $\mathrm{NH}_{4} \mathrm{OH}(28 \%, 10 \mathrm{~mL})$ and 1 part of $\mathrm{H}_{2} \mathrm{O}_{2}(30 \%, 10 \mathrm{~mL})$ and then heated to $70{ }^{\circ} \mathrm{C}$. During heating at a constant temp of $70^{\circ} \mathrm{C}$, the ITO substrate was dipped for $15 \mathrm{~min}$ and then rinsed twice in the water. Lastly, to remove the water, the substrate was rinsed with IPA and dried in a heating chamber. After the ozone-cleaning with a deep-UV light, the substrate was transferred to the spin-cast chamber for spin-coating. Colloidal inks of $\mathrm{ZnO}(20 \mathrm{mg} / \mathrm{mL})$, Si QDs $(10 \mathrm{mg} / \mathrm{mL})$, and $\mathrm{WO}_{3}(5 \mathrm{mg} / \mathrm{mL})$ nanocrystals were spin-casted sequentially with rotation speeds of 2000, 1000, and $1000 \mathrm{rpm}$, respectively. The colloidal ink of $\mathrm{ZnO}$ nanocrystals was prepared by following the procedure reported previously [24]. We used ethanol, toluene, and IPA to prepare colloidal inks of $\mathrm{ZnO}, \mathrm{Si} \mathrm{QD}$, and $\mathrm{WO}_{3}$, respectively. The use of the orthogonal solvents enabled the underlying layer to avoid any damage caused by spin-coating over layers. In each step of coating, the films were dried for $1 \mathrm{~h}$ at $120^{\circ} \mathrm{C}$ in a vacuum chamber. The film thicknesses of $\mathrm{ZnO}$ and $\mathrm{WO}_{3}$ were approximately 22 and $13 \mathrm{~nm}$ respectively. The top electrode $\mathrm{Al}$ of $150 \mathrm{~nm}$ was deposited using a mask by the thermal evaporation technique.

\subsection{Characterization}

To avoid the solvent effect on PL QYs, optical properties were measured using the QD films deposited onto quartz glass substrates. The PL spectrum was measured by a modular double grating Czerny-Turner monochromator and iHR 320 monochromator (1200 lines/mm of gratings) coupled with a photomultiplier tube (PMT-955) mounted on fluorescence spectrometer (NanoLog Horiba JovinYvon, Kyoto, Japan). A $450 \mathrm{~W}$ xenon lamp was used for photoexcitation. The spectral resolution of the spectrofluorometer was $\sim 0.3 \mathrm{~nm}$. The absolute PL QYs were measured at room temperature using the QY measurement system (C11347-11, Hamamatsu Photonics Co. Ltd., Shizuoka, Japan). A $150 \mathrm{~W}$ xenon lamp for excitation was mounted on the system. A monochromator for wavelength discrimination, an integrating sphere for a sample chamber, and a multichannel analyzer for signal detection were coupled to the system.

I-V characterization of the device was investigated by a computer programmable Keithley 2400 and 2425. To measure the Si-QLED characteristics, the prototype QLED was kept just above a silicon photodiode to sense the emitted light from the QLED. Photodiode current was measured by the Keithley 2425. EL spectra were measured by collecting the light by an objective lens equipped with a SMA905 connector and sent by optical fiber $(\phi=1000 \mu \mathrm{m})$ to a Si CCD array multichannel monochromator 
with 2048 pixels (OP-FLAME-S-OW, Ocean Photonic, Ltd., Tokyo, Japan). EQE was calculated as a ratio of the number of forward-emitted photons to the number of injected electrons, expressed as:

$$
\mathrm{EQE}[\%]=N_{\text {phot }^{*}|e| I_{\mathrm{d}}} \times g \times 100
$$

where $N_{\text {phot }}$ is the number of forward-emitted photons which is counted by the photodiode, $I_{\mathrm{d}}$ is the current passing through the device under operation voltage, and $|e|$ is the magnitude of charge of an electron.

The geometric factor, $g$, is expressed as:

$$
g=\left(a^{2}+L^{2}\right) / a^{2}
$$

where $a$ is the length and breadth of a square photodiode and $L$ is the distance between the emitting QLED pixel and the photodiode.

The photodiode current, divided by the responsivity value of the photodiode at the peak energy of the EL spectrum, gives the output photon for light from the device. The number of the photon is calculated by simply dividing with $\mathrm{hc} / \lambda$. The formula for the EQE calculation is simplified as expressed:

$$
\mathrm{EQE}(\%)=\frac{I(\text { Photodiode }) \times \lambda(\text { peak }) \times \text { electron charge } \times g \times 100}{R(\lambda) \times h c \times I(\text { device current })}
$$

where I (photodiode) is the photo current, in which dark current is subtracted, detected by a photodiode (Hamamatsu S1336-8BQ, Shizuoka, Japan) placed just below the EL device. I (device) is the device current, when the device is not operative. $R$ is the responsivity of the photodiode and $g$ is the configuration factor of our measurement set-up, which we estimated was 4 .

Luminance $\left(\mathrm{Cd} / \mathrm{m}^{2}\right)$ was estimated using the value of EQE and the area of the EL spectrum, as expressed:

$$
\text { Luminance }(\mathrm{Cd} / \mathrm{m} 2)=683.002 \times \text { EL area under the curve Normalized } \times C I E \times I \text { (device }) \times E Q E
$$

\section{Results and Discussion}

Figure $1 b, c$ schematically illustrate the inverted QLED (i.e., ITO/ZnO/Si/ $\mathrm{WO}_{3} / \mathrm{Al}$ ) as well as its flat energy band diagram. In contrast to a conventional structure, the electrons were injected from the ITO electrode while the holes were injected from the Al electrode in the inverted device structure, as indicated by arrows in Figure $1 \mathrm{~b}$. With an electron affinity of $4.3 \mathrm{eV}$ and an ionization potential of $7.6 \mathrm{eV}$ [25], the $\mathrm{ZnO}$ EIL/ETL worked for efficient electron injection from the ITO electrode into an active layer of $\mathrm{Si}$ QDs. The layer of $\mathrm{ZnO}$ also worked for confinement of holes within the active layer because of the valence band offset at the $\mathrm{ZnO} / \mathrm{QD}$ interface. Both effects strengthened the carrier recombination efficiency. On the other hand, the $\mathrm{WO}_{3} \mathrm{HIL} / \mathrm{HTL}$ provided a deep level of the highest occupied molecular orbital (HOMO) $(7 \mathrm{eV})$ which was helpful for hole injection in the HOMO level of the Si QD (5.5 eV) [26].

Electrical characterization of the QLED is provided in Figure 2. I-V characteristics of the device and photodiode have been plotted in Figure 2a. The QLED showed good rectification behavior. Current density-voltage $(\mathrm{J}-\mathrm{V})$ characteristics were replotted in a log-log scale in Figure $2 \mathrm{~b}$. The experimental data was linearly fitted to reveal the power law relation $J \propto V^{n}$ where the value of $n$ was a signature of the conduction mechanism. In Figure $2 b, J-V$ characteristics were fitted with two linear functions. The first one was seen in a low voltage region $(0-0.5 \mathrm{~V})$, while another one was seen in a high voltage region $(0.5-3 \mathrm{~V})$. In the low voltage region, the relation was $\mathrm{J}_{\mathrm{V}} \mathrm{V}^{1.9}$, indicative of trap free space-charge-limited conduction (SCL). The SCL current appeared at the same time as the injected carrier density exceeded the density of carriers produced by thermal excitation. In the absence of any traps for carriers, the ideal SCL current density is given by the Mott-Gurney Law, $\mathrm{J}^{2} \mathrm{~V}^{2}[6]$. While in the high voltage range, 
the value of exponent (at high current) increased and the relation became $J \propto V^{5.2}$ which indicated the appearance of the SCLC mechanism with exponential distribution of traps [16,27]. The increase in the J-V slope at $\sim 3 \mathrm{~V}$ coincided with the photodiode attached just below the active device giving the response when the Si-QLED started emitting light. So, the estimated value of turn-on voltage was as low as $3 \mathrm{~V}$.
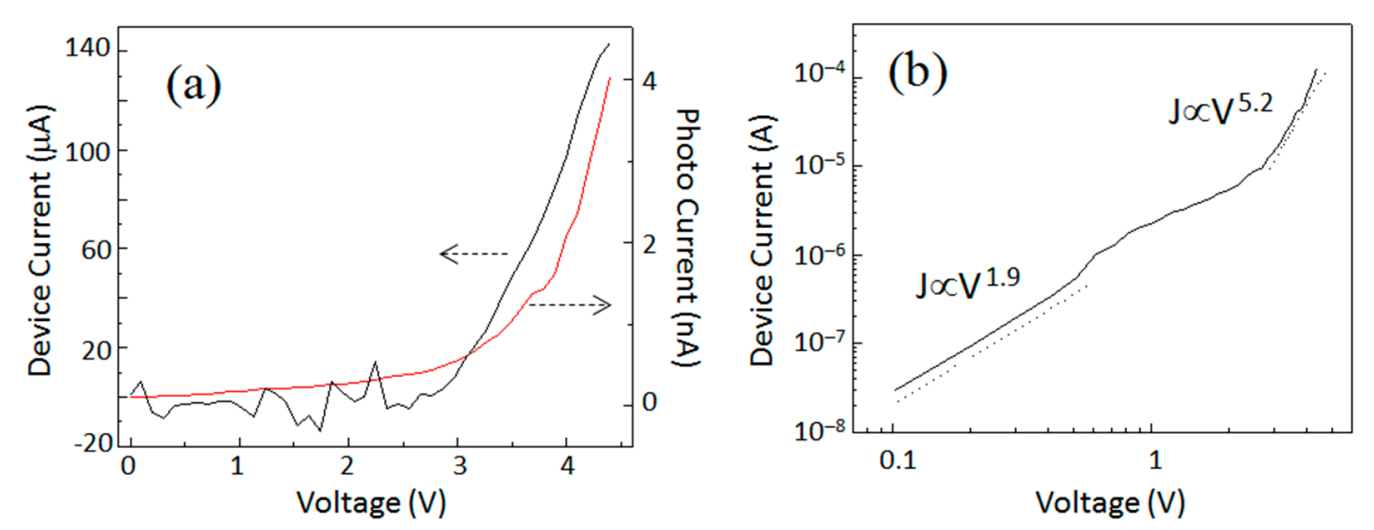

Figure 2. (a) Device I-V characteristics (black-curve) and photodiode I-V characteristics (red-curve) and (b) I-V in double log scale to fit linearly with a different slope (as guided by the dotted line).

The values of EQE for our Si-QLEDs are plotted in Figure 3a as a function of the injected current density. EQE reached a maximum value of $0.25 \%$ at a current density $0.00125 \mathrm{~A} / \mathrm{cm}^{2}$. With increasing the device current density more, the EQE decreased in a small decrement. In the present device, the EQE value was low compared to the hybrid Si-QLED device. A possible reason for such a low value of EQE might be discussed as a result of the high energy barrier between the QD layer and the $\mathrm{WO}_{3}$ HIL/HTL. The steeply pitched slope in energy difference between each layer should be smoothed by adding other layers for efficient transportation of holes without loss of energy. From the flat band energy diagram of this device structure, the band alignment of each layer was far from optimum, which allowed for a smooth charge injection from the one layer to the next layer. Further improvement of the band alignment of the layers for smooth flowing of electrons and holes will be the focus in our next work. In Figure 3b, we compared PL and EL spectra in terms of peak position and spectral linewidth. Clearly, the EL emission peak was observed at $680 \mathrm{~nm}$. The full- width at half- maximum (FWHM) of the EL spectrum was as narrow as $130 \mathrm{~nm}$ of the corresponding PL spectral FWHM. Furthermore, there was no broadening or shift of the EL spectrum with respect to the PL spectrum. The nearly-overlapped spectral shape and position suggested that the emission from the QLED originated from the carrier recombination across the fundamental energy gap in the QD. We observed a vivid red-light emission by naked- eye when the QLED was operated at $5 \mathrm{~V}$ (see Figure 3c). Figure 3d plots the typical luminance curves as a function of the current density. The luminance reached a value as high as $1400 \mathrm{~cd} / \mathrm{m}^{2}$ at current density $0.0032 \mathrm{~A} / \mathrm{cm}^{2}$. 

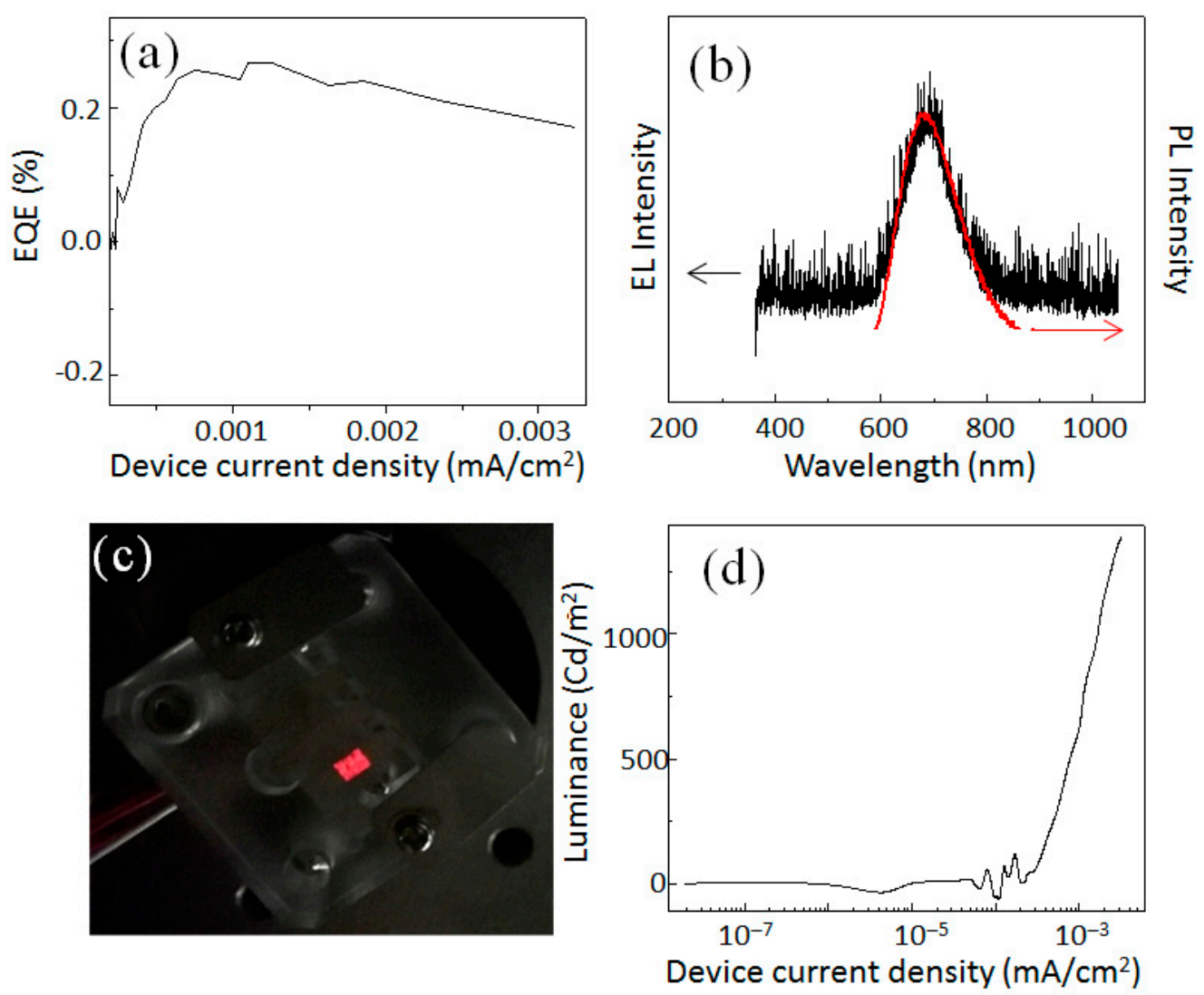

Figure 3. (a) EQE-current density, (b) a typical EL spectrum (PL spectrum of the corresponding decane-terminated Si QD), (c) a typical digital photograph demonstrating a representative red-light emitting Si-QLED, and (d) luminescence versus device current density.

Blueshift of EL spectra with increasing device bias is still one of common problems observed for QLEDs prepared from colloidal inks of semiconductors [20]. For Si, a similar problem is reported in literature when the QLED has a conventional device structure. For example, Liu et al. reported $\sim 50 \mathrm{~nm}$ blueshift of the EL peak at a $6.5 \mathrm{~V}$ of operation voltage [7]. Maier-Flaig et al. reported that the EL peak shifted $\sim 15 \mathrm{~nm}$ toward a shorter wavelength when the applied voltage increased from 3.5 to $10 \mathrm{~V}$ [28]. Blueshift of EL peak with increasing driving voltage has been also reported for NIR-emitting Si-QLEDs $[4,29]$. It is reported that inversed device structures of QLEDs work for minimizing such unwanted spectral shift even at high driving voltage [20,30,31]. The inverted device structures reported usually consist of an organic-inorganic multilayer thin film architecture which is developed based on ITO/ZnO/QDs/CBP/Al structure. In this work, the CBP layer working as organic HIL/HTL was replaced by an inorganic $\mathrm{WO}_{3}$ layer to widen the applicability of the inverted device architecture. As clearly shown in Figure 4a, the EL peak did not shift in the applied voltage range between 4 and $13 \mathrm{~V}$. This implies that color purity is high with respect to the applied voltage to the device. In spite of the recent progress in Si-QLEDs, achieving a superior stability, the device in a high humidity environment is still a challenging theme. To investigate the stability of our Si-QLED against humidity, the device EQE value was measured after long time exposure. EQE values as a function of device current density were continuously measured for the device (see Figure $4 \mathrm{~b}$ ). The black curve shows the EQE trend with increasing device current density for the as-prepared QLED. After the measurement, the device was continuously exposed to high humidity conditions ( 80\%) in ambient air for 15 and 45 days without any kind of encapsulation. Then, the values of EQE were measured for the same device and compared. As summarized in Figure $4 \mathrm{~b}$, it is obvious that the values of EQE were not diminishing greatly even after exposure to $80 \%$ humidity in air. Specifically, EQE values of the same devices after the exposure 
dropped a maximum $10 \%$ when compared to the value of the as-prepared device. Furthermore, we see similar decreasing trends of EQE with increasing current density for the device. EL spectra were also measured. As expected, the EL peak positions were also unchanged even after the exposure to moisture in air. The stability against humidity may be improved when compared to the hybrid QLED device consisting an organic layer (i.e., PEDOT:PSS), which is less stable in a high humidity environment. In our case, since the multilayer is completely composed of inorganic nanocrystals, the resultant QLED could be much more stable even with long exposure in the high humidity environment.
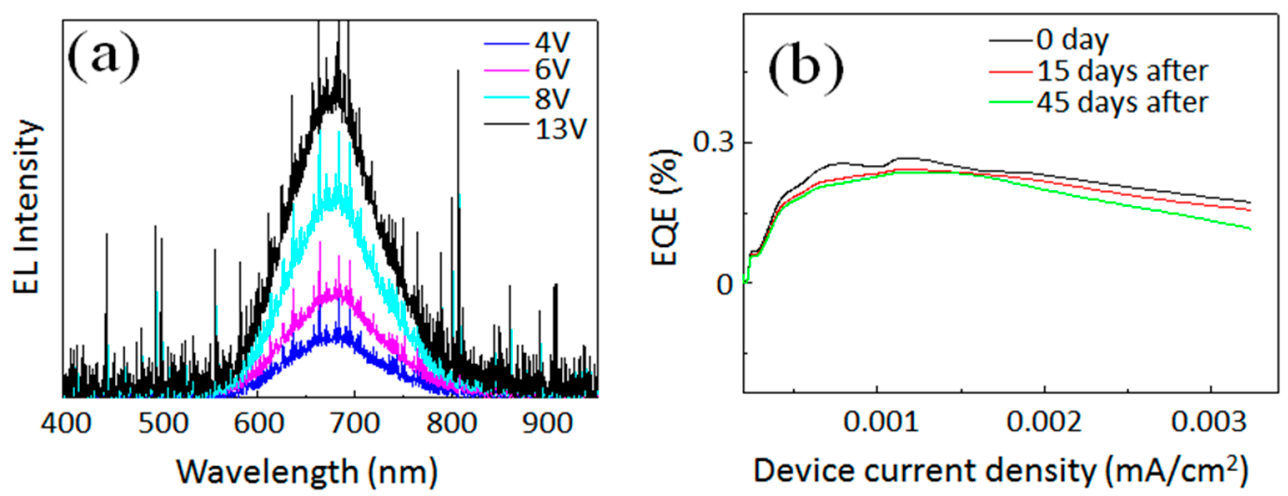

Figure 4. (a) Voltage variance of the EL spectra at different applied bias and (b) EQE value with device current density for three Si-QLEDs stored at 80\% humidity in ambient air.

\section{Conclusions}

We demonstrated an inverted device structure of a Si-QLED which exhibited a high stability against humidity in ambient air. The device consisted of inorganic multilayers where $\mathrm{ZnO}$ EIL/ETL and $\mathrm{WO}_{3} \mathrm{HIL} / \mathrm{HTL}$ sandwiched an optically-active layer of red-emitting Si QDs. The multilayered structure was prepared by a spin-coating technique, except for the $\mathrm{Al}$ electrode. Based on the carrier transition in the inverted device structure, electrons were injected from an ITO electrode while holes were injected from an $\mathrm{Al}$ electrode. The J-V characteristic indicated the carrier recombination in the QD layer. We observed a good EL performance for the Si-QLEDs, which were exposed to $80 \%$-humidity for 45 days in ambient air. The results obtained in this work encourage us to push forward the development and practical use of all-inorganic QLEDs.

Author Contributions: B.G. and N.S. designed research, B.G. performed research, and B.G and N.S. wrote the paper.

Funding: A-step (JPMJTS1619) from Japan Science and Technology Agency and the Izumi Science and Technology Foundation (2018-J-053). Kakenhi (19K22176), B.G. thanks to the JSPS international fellowship program for research in Japan (L18530).

Conflicts of Interest: The authors declare no conflict of interest.

\section{References}

1. Chinnathambi, S.; Shirahata, N. Recent Advances on Fluorescent Biomarkers of Near-Infrared Quantum Dots for In-Vitro and in-Vivo Imaging. Sci. Technol. Adv. Mater. 2019, 20, 337-355. [CrossRef] [PubMed]

2. Yong, K.; Law, W.; Hu, R.; Ye, L.; Liu, L.; Swihart, M.T.; Prasad, P.N. Nanotoxicity Assessment of Quantum Dots: From Cellular to Primate Studies. Chem. Soc. Rev. 2013, 42, 1236-1250. [CrossRef] [PubMed]

3. Chandra, S.; Ghosh, B.; Beaune, G.; Nagarajan, U.; Yasui, T.; Nakamura, J.; Tsuruoka, T.; Baba, Y.; Shirahata, N.; Winnik, F.M. Functional Double-Shelled Silicon Nanocrystals for Two-Photon Fluorescence Cell Imaging: Spectral Evolution and Tuning. Nanoscale 2016, 8, 9009-9019. [CrossRef] [PubMed]

4. Cheng, K.-Y.; Anthony, R.; Kortshagen, U.R.; Holmes, R.J. High-Efficiency Silicon Nanocrystal Light-Emitting Devices. Nano Lett. 2011, 11, 1952-1956. [CrossRef] [PubMed] 
5. Puzzo, D.P.; Henderson, E.J.; Helander, M.G.; Wang, Z.; Ozin, G.A.; Lu, Z. Visible Colloidal Nanocrystal Silicon Light-Emitting Diode. Nano Lett. 2011, 11, 1585-1590. [CrossRef] [PubMed]

6. Ghosh, B.; Masuda, Y.; Wakayama, Y.; Imanaka, Y.; Inoue, J.; Hashi, K.; Deguchi, K.; Yamada, H.; Sakka, Y.; Ohki, S.; et al. Hybrid White Light Emitting Diode Based on Silicon Nanocrystals. Adv. Funct. Mater. 2014, 24, 7151-7160. [CrossRef]

7. Liu, X.; Zhao, S.; Gu, W.; Zhang, Y.; Qiao, X.; Ni, Z.; Pi, X.; Yang, D. Light-Emitting Diodes based on Colloidal Silicon Quantum Dots with Octyl and Phenylpropyl Ligands. ACS Appl. Mater. Interf. 2018, 10, 5959-5966. [CrossRef]

8. Yamada, H.; Shirahata, N. Silicon Quantum Dot Light Emitting Diode at $620 \mathrm{~nm}$. Micromachines 2019, 10, 318. [CrossRef]

9. Cao, F.; Wang, S.; Wang, S.; Wu, Q.; Zhao, D.; Yang, X. A Layer-by-Layer Growth Strategy for Large-Size InP/ZnSe/ZnSCore-Shell Quantum Dots Enabling High-Efficiency Light-Emitting Diodes. Chem. Mater. 2018, 30, 8002-8007. [CrossRef]

10. Kolny-Olesiak, J.; Weller, H. Synthesis and Application of Colloidal CuInS 2 Semiconductor Nanocrystals. ACS Appl. Mater. Interf. 2013, 5, 12221-12237. [CrossRef]

11. Shirasaki, Y.; Supran, G.J.; Bawendi, M.G.; Bulović, V. Emergence of Colloidal Quantum-Dot Light-Emitting Technologies. Nat. Photo. 2013, 7, 13-23. [CrossRef]

12. Ghosh, A.P.; Gerenser, L.J.; Jarman, C.M.; Fornalik, J.E. Thin-film Encapsulation of Organic Light-Emitting Devices. Appl. Phys. Lett. 2005, 86, 223503. [CrossRef]

13. Murase, S.; Yang, Y. Solution Processed $\mathrm{MoO}_{3}$ Interfacial Layer for Organic Photovoltaics Prepared by a Facile Synthesis Method. Adv. Mater. 2012, 24, 2459-2462. [CrossRef] [PubMed]

14. Jasieniak, J.J.; Seifter, J.; Jo, J.; Mates, T.; Heeger, A.J. A Solution-Processed MoOx Anode Interlayer for Use within Organic Photovoltaic Devices. Adv. Funct. Mater. 2012, 22, 2594-2605. [CrossRef]

15. Maier-Flaig, F.; Kübel, C.; Rinck, J.; Bocksrocker, T.; Scherer, T.; Prang, R.; Powell, A.K.; Ozin, G.A.; Lemmer, U. Looking Inside a Working SiLED. Nano Lett. 2013, 13, 3539-3545. [CrossRef] [PubMed]

16. Caruge, J.-M.; Halpert, J.E.; Bulović, V.; Bawendi, M.G. NiO as an Inorganic Hole-Transporting Layer in Quantum-Dot Light-Emitting Devices. Nano Lett. 2006, 6, 2991-2994. [CrossRef] [PubMed]

17. Caruge, J.M.; Halpert, J.E.; Wood, V.; Bulović, V.; Bawendi, M.G. Colloidal Quantum-Dot Light-Emitting Diodes with Metal-Oxide Charge Transport Layers. Nat. Photon. 2008, 2, 247-250. [CrossRef]

18. Wood, V.; Panzer, M.J.; Halpert, J.E.; Caruge, J.-M.; Bawendi, M.G.; Bulovic, V. Selection of Metal Oxide Charge Transport Layers for Colloidal Quantum Dot LEDs. ACS Nano 2009, 3, 3581-3586. [CrossRef]

19. Shi, Z.; Li, Y.; Zhang, Y.; Chen, Y.; Li, X.; Wu, D.; Xu, T.; Shan, C.; Du, G. High-Efficiency and Air-Stable Perovskite Quantum Dots Light-Emitting Diodes with an All-Inorganic Heterostructure. Nano Lett. 2016, 17, 313-321. [CrossRef]

20. Ghosh, B.; Yamada, H.; Chinnathambi, S.; Özbilgin, I.N.G.; Shirahata, N. Inverted Device Architecture for Enhanced Performance of Flexible Silicon Quantum Dot Light-Emitting Diode. J. Phys. Chem. Lett. 2018, 9 , 5400-5407. [CrossRef]

21. Chandra, S.; Masuda, Y.; Shirahata, N.; Winnik, F.M. Transition Metal Doped NIR Emitting Silicon Nanocrystals. Angew. Chem. Int. Ed. 2017, 56, 6157-6160. [CrossRef] [PubMed]

22. Ghosh, B.; Takeguchi, M.; Nakamura, J.; Nemoto, Y.; Hamaoka, T.; Chandra, S.; Shirahata, N. Origin of the Photoluminescence Quantum Yields Enhanced by Alkane-Termination of Freestanding Silicon Nanocrystals: Temperature-Dependence of Optical Properties. Sci. Rep. 2016, 6, 36951. [CrossRef] [PubMed]

23. Ghosh, B.; Hamaoka, T.; Nemoto, Y.; Takeguchi, M.; Shirahata, N. Impact of Anchoring Monolayers on the Enhancement of Radiative Recombination in Light-Emitting Diodes Based on Silicon Nanocrystals. J. Phys. Chem. C 2018, 122, 6422-6430. [CrossRef]

24. Stouwdam, J.W.; Rene', J.A.J. Red, Green, and Blue Quantum Dot LEDs with Solution Processable ZnO Nanocrystal Electron Injection Layers. J. Mater. Chem. 2008, 18, 1889-1894. [CrossRef]

25. Brown, P.R.; Lunt, R.R.; Zhao, N.; Osedach, T.P.; Wanger, D.D.; Chang, L.; Bawendi, M.G.; Bulović, V. Improved Current Extraction from $\mathrm{ZnO} / \mathrm{PbS}$ Quantum Dot Heterojunction Photovoltaics using a $\mathrm{MoO}_{3}$ Interfacial Layer. Nano Lett. 2011, 11, 2955-2961. [CrossRef]

26. Tan, Z.; Li, L.; Cui, C.; Ding, Y.; Xu, Q.; Li, S.; Qian, D.; Li, Y. Solution-Processed Tungsten Oxide as an Effective Anode Buffer Layer for High-Performance Polymer Solar Cells. J. Phys. Chem. C 2012, 116, 18626-18632. [CrossRef] 
27. Hikmet, R.A.M.; Talapin, D.V.; Weller, H. Study of Conduction Mechanism and Electroluminescence in CdSe/ZnS Quantum Dot Composites. J. Appl. Phys. 2003, 93, 3509-3514. [CrossRef]

28. Maier-Flaig, F.; Rinck, J.; Stephan, M.; Bocksrocker, T.; Bruns, M.; Kübel, C.; Powell, A.K.; Ozin, G.A.; Lemmer, U. Multicolor Silicon Light-Emitting Diodes (SiLEDs). Nano Lett. 2013, 13, 475-480. [CrossRef]

29. Cheng, K.-Y.; Anthony, R.; Kortshagen, U.R.; Holmes, R.J. Hybrid Silicon Nanocrystal-Organic Light-Emitting Devices for Infrared Electroluminescence. Nano Lett. 2010, 10, 1154-1157. [CrossRef]

30. Kwak, J.; Bae, W.K.; Lee, D.; Park, I.; Lim, J.; Park, M.; Cho, H.; Woo, H.; Yoon, D.Y.; Char, K.; et al. Bright and Efficient Full-Color Colloidal Quantum Dot Light-Emitting Diodes Using an Inverted Device Structure. Nano Lett. 2012, 12, 2362-2366. [CrossRef]

31. Wang, L.; Lv, Y.; Lin, J.; Fan, Y.; Zhao, J.; Wang, Y.; Liu, X. High-Efficiency Inverted Quantum Dot Light-Emitting Diodes with Enhanced Hole Injection. Nanoscale 2017, 9, 6748-6754. [CrossRef] [PubMed]

(C) 2019 by the authors. Licensee MDPI, Basel, Switzerland. This article is an open access article distributed under the terms and conditions of the Creative Commons Attribution (CC BY) license (http://creativecommons.org/licenses/by/4.0/). 